\title{
Revisiting the Real Graviton Effects at CERN LHC within the Quantum Gravity Theory with Large Extra Dimensions
}

\author{
Xing-Gang $\mathrm{Wu}^{*}$ and Zhen-Yun Fang \\ Department of Physics, Chongqing University, Chongqing 400044, P.R. China
}

\begin{abstract}
CERN LHC provides a good experimental platform to perturbatively probe the fundamental gravity scale up to several $\mathrm{TeV}$, with the precise value depending on the number of extra dimensions. The leading experimental signal of graviton at LHC is from the process $p p \rightarrow j e t+\mathbb{E}_{T}$, where $\mathbb{F}_{T}$ stands for the transverse missing energy. A detailed discussion on the hadronic production of real graviton through hard subprocesses: $q \bar{q} \rightarrow G+g, g+q \rightarrow G+q$ and $g+g \rightarrow G+g$ have been studied within the quantum gravity theory with large extra dimensions. The main theoretical uncertainties together with the dominant standard model background to these processes, e.g. $q \bar{q} \rightarrow Z^{0}+g$ and $g+q \rightarrow Z^{0}+q$ with $Z^{0}$ further decaying into neutrinos, have also been discussed. It is found that only in certain jet energy region and with certain number of extra dimensions can the quantum gravity signal be distinguished from the background, which inversely lead to the effective scale $M_{D}$ to be probed up to $(8.8 \pm 0.9) \mathrm{TeV}$ for two extra dimensions, and $(5.9 \pm 0.5)$ $\mathrm{TeV}$ for four extra dimensions with sufficient integrated luminosity, e.g. $100 \mathrm{fb}^{-1}$, at CERN LHC.
\end{abstract}

PACS numbers: 04.50.-h, 04.60.-m, 04.60.Bc, 12.38.Aw

Keywords: quantum gravity, large extra dimension, uncertainty estimation

* email: wuxg@cqu.edu.cn 


\section{INTRODUCTION}

It has been purposed that standard model (SM) particles live in the usual $3+1$ dimensional space, while gravity can propagate in a higher-dimension space [1, 2]. Such a scenario is helpful to reduce the fundamental mass scale from the large Plank scale down to be about $T e V$-scale and then to solve the so-called hierarchy problem, so it arouses people's interests since its first announcement. Numerous attempts have been carried out to find the signal of the large extra dimension, i.e. measurements of gravity at short distances, studies of various astrophysical and cosmological implications of large extra dimension and collider searches for virtual and real graviton effects [3, 4, 5, 6, 7, 8, 9, 10]. The CERN LHC, with its high collision energy $(14 \mathrm{TeV})$ and high luminosity $\left(10^{34} \mathrm{~cm}^{-2} \mathrm{~s}^{-1}\right)$, shall provide a better platform to study the extra-dimension phenomenology both experimentally and theoretically.

The leading experimental signal of real graviton at LHC is from the hadronic process $p p \rightarrow j e t+\mathbb{E}_{T}$ with $\mathbb{E}_{T}$ stands for the transverse missing energy. We shall present a detailed discussion on the hadronic production of graviton through the hard subprocesses: $q \bar{q} \rightarrow$ $G+g, g+q \rightarrow G+q$ and $g+g \rightarrow G+g$. By using the quantum gravity effective theory with large extra dimensions [11, 12], we make a try to study the quantum gravity effects and its dependence on the number of extra dimension, and to study up to what energy scale can LHC probe. Furthermore, main theoretical uncertainty for the graviton production shall be studied, which includes the parton distribution functions (PDFs) for the initial partons, the choice of the factorization scale $\mu_{F}^{2}$ and the typical energy scale $Q^{2}$ for the hard scattering amplitude, the number of the extra dimension for the graviton production, and etc..

The processes $q \bar{q} \rightarrow g+Z^{0}$ and $q g \rightarrow q Z^{0}$, followed by an invisible decay of $Z^{0}$, i.e. $Z^{0} \rightarrow \nu \bar{\nu}$, give an irreducible physical background to graviton production. We refer to these processes as the 'SM background'. We will estimate the observability of the graviton signal by comparing its hadronic cross-section to that of 'SM background'. There are other important background sources from miss-measured jets and $W$ production with forward leptons, however as argued in Ref.[13] that these backgrounds decrease sharply as the lower bound on missing $E_{T}$ is increased, so we shall not considered it here.

The remainder of the paper is organized as follows. Section II is devoted to give the main formulae for graviton production within the framework of the quantum gravity effective 
theory with large extra dimensions. Numerical results and discussions are presented in Section III, where the uncertainties in estimates and a discussion on the value of the effective energy scale $M_{D}$ shall be presented. The last section is reserved for a summary.

\section{CALCULATION TECHNOLOGY}

According to the QCD factorization formulae, the hadronic production of graviton at the collision center of mass energy $\sqrt{S}$ can generally be written as

$$
\begin{aligned}
& d \sigma\left(S, E_{T}, \cdots\right)= \\
& \sum_{i j} \iint d x_{1} d x_{2} F_{H_{1}, P_{1}}^{i}\left(x_{1}, \mu_{F}^{2}\right) F_{H_{2}, P_{2}}^{j}\left(x_{2}, \mu_{F}^{2}\right) d \hat{\sigma}_{i j \rightarrow j e t+\mathbb{H}_{T}}\left(P_{1}, P_{2}, x_{1}, x_{2}, \mu_{F}^{2}, Q^{2}, \hat{s}, \cdots\right),
\end{aligned}
$$

where $F_{H_{1}, P_{1}}^{i}\left(x_{1}, \mu_{F}^{2}\right)$ and $F_{H_{2}, P_{2}}^{j}\left(x_{2}, \mu_{F}^{2}\right)$ are PDFs of incoming hadrons $H_{1}$ (momentum $P_{1}$ ) and $H_{2}$ (momentum $P_{2}$ ) for parton $i$ (with the momentum fraction $x_{1}$ ) and parton $j$ (with the momentum fraction $x_{2}$ ) respectively. $Q^{2}$ is the 'characteristic energy scale of the subprocess squared'; and $\mu_{F}^{2}$ is the 'energy scale squared' where the factorization about the PDFs and the hard subprocess is made. Usually for leading order (LO) calculation to obtain the best results, the two scales $\mu_{F}^{2}$ and $Q^{2}$ are carried out as the same, thus later on we take $\mu_{F}^{2}=Q^{2}$ except one case when estimating the uncertainty from LO and the ambiguity of the choices about $\mu_{F}^{2}$ and $Q^{2}$.

$d \hat{\sigma}_{i j \rightarrow j e t+E_{T}}$ stands for the differential cross-section of the relevant hard subprocess, in which $\hat{s}=x_{1} x_{2} S$ is the center of mass energy of the subprocess and $\mathbb{F}_{T}$ stands for the missing transverse energy. Within the framework of the quantum gravity theory with large extra dimensions, the differential cross-section for inclusive graviton $(G)$ production, i.e. $i j \rightarrow G k$ with $i, j$ and $k$ stands for corresponding partons, can be written as [12]

$$
\frac{d^{2}}{d t d m} \hat{\sigma}_{i j \rightarrow G k}=S_{\delta-1} \frac{\bar{M}_{P}^{2}}{M_{D}^{2+\delta}} m^{\delta-1} \frac{d \hat{\sigma}_{m}}{d t}
$$

where $d \hat{\sigma}_{m} / d t$ stands for the differential cross-section for producing a single Kaluza-Klein graviton of mass $m, \bar{M}_{P}=M_{P} / \sqrt{8 \pi}=2.4 \times 10^{18} \mathrm{GeV}, S_{\delta-1}$ is the surface of a unit-radius sphere in $\delta$ dimensions, for $\delta=2 n$ and $n$ integer, $S_{\delta-1}=2 \pi^{n} /(n-1)$ ! and for $\delta=2 n+1$,

$S_{\delta-1}=2 \pi^{n} / \Pi_{k=0}^{n-1}\left(k+\frac{1}{2}\right)$. For the present $2 \rightarrow 2$ subprocesses, the Mandelstam variables are defined as $s=\left(p_{i}+p_{j}\right)^{2}, t=\left(p_{i}-p_{G}\right)^{2}$ and $u=\left(p_{i}-p_{k}\right)^{2}$. Since the graviton interaction vertex is suppressed by $1 / \bar{M}_{P}$, it can be found that $\hat{\sigma}_{m} \propto \bar{M}_{P}^{-2}$, and the factor $\bar{M}_{P}^{2}$ appearing 
from the phase-space summation exactly cancels the Planck mass dependence in Eq. (2). In another words, the large phase space of the Kaluza-Klein modes, corresponding to the large volume of the compactified space, exactly cancels the dependence on $\bar{M}_{P}$ and gives an effective interaction suppressed only by inverse powers of $M_{D}$. This is the reason why sizable contributions from the graviton may be observed at LHC, and inversely, one can estimate to what energy scale can LHC probe. For the differential partonic cross-sections $d \hat{\sigma}_{m} / d t$ that produce a single Kaluza-Klein graviton with mass $m$, we obtain

$$
\begin{aligned}
\frac{d \hat{\sigma}_{m}}{d t}(q \bar{q} \rightarrow g G) & =\frac{\alpha_{s}\left(Q^{2}\right)}{36 s \bar{M}_{P}^{2}} F_{1}(t / s, u / s), \\
\frac{d \hat{\sigma}_{m}}{d t}(q g \rightarrow q G) & =\frac{\alpha_{s}\left(Q^{2}\right)}{96 s \bar{M}_{P}^{2}} F_{2}(t / s, u / s), \\
\frac{d \hat{\sigma}_{m}}{d t}(g g \rightarrow g G) & =\frac{3 \alpha_{s}\left(Q^{2}\right)}{16 s \bar{M}_{P}^{2}} F_{3}(t / s, u / s),
\end{aligned}
$$

where $Q^{2}$ stands for the characteristic energy scale of the hard scattering amplitude, $q$ stands for the light quark $u, d$ and $s$ respectively, and the functions $F_{1,2,3}$ take the following form

$$
\begin{aligned}
& F_{1}(x, y)=\frac{1}{x y}(4 x y+z)\left(1-2 x y+z^{2}\right), \\
& F_{2}(x, y)=\frac{1}{x z}\left(2 x-z^{2}-y^{2}\right)\left(z+z^{2}+x(4+z)\right), \\
& F_{3}(x, y)=\frac{1}{x z}\left[x^{2} y^{2}+2 x y\left(z^{2}-z+1\right)+\left(1+z+z^{2}\right)^{2}\right] .
\end{aligned}
$$

The relation $z=1+x+y$ is implicitly adopted that can be deduced from the fact of $s+t+u=m^{2}$. It can be easily find that $F_{1,3}(x, y)=F_{1,3}(y, x)$ as is the requirement of the invariance under exchange of the Mandelstam variables $t$ and $u$.

For the background subprocesses $i j \rightarrow Z^{0} k$, we obtain

$$
\begin{aligned}
& \frac{d \hat{\sigma}}{d t}\left(q \bar{q} \rightarrow g Z^{0}\right)=\frac{\pi \alpha\left(Q^{2}\right) \alpha_{s}\left(Q^{2}\right)\left(L_{q}^{2}+R_{q}^{2}\right)}{9 x_{W}\left(1-x_{W}\right) s^{2} t u}\left(m_{Z^{0}}^{4}+s^{2}-2 t u\right), \\
& \frac{d \hat{\sigma}}{d t}\left(q g \rightarrow q Z^{0}\right)=\frac{\pi \alpha\left(Q^{2}\right) \alpha_{s}\left(Q^{2}\right)\left(L_{q}^{2}+R_{q}^{2}\right)}{18 x_{W}\left(1-x_{W}\right) m_{Z^{0}}^{2} s^{3} t}\left[6 s m_{Z^{0}}^{4}-2 s(s+t) m_{Z^{0}}^{2}+t^{3}\right],
\end{aligned}
$$

where $x_{W}=\sin ^{2} \theta_{W}, R_{q}=-2 e_{q} x_{W}$ and $L_{q}=1 / 2-2 e_{q} x_{W}$ with $e_{q}$ stands for the electric charge of $q$-quark.

\section{NUMERICAL RESULTS AND DISCUSSIONS}

In the following, we shall first discuss the uncertainties in estimating the graviton $/ Z^{0}$ production. For the LO estimation, we shall concentrate our attention on the main uncer- 
tainties, which include the PDFs for the light quarks, the choice of the factorization scale $\mu_{F}$ and the typical energy scale $Q^{2}$ for the hard scattering amplitude and the number of the extra dimension for the graviton production.

The number of extra dimension $(\delta)$ can either be too small or too large. We are interested in the case in which $\delta$ is not too large (say $\delta \lesssim 6$ ), under such condition the mass splitting $\Delta m$ is so small that the sum over the different Kaluza-Klein states can be replaced by a continuous integration, and then the enormous number of accessible Kaluza-Klein modes can rightly compensate the $1 / \bar{M}_{P}^{2}$ factor in the scattering amplitude. While an even larger number of extra dimensions shall lead to the mass splitting $\Delta m$ become comparable with the experimental energy resolution, hence only a smaller number of Kaluza-Klein modes can be produced, and then the total cross-section is negligible due to the unavoidable $1 / \bar{M}_{P}^{2}$ suppression. On the other hand, it is argued that $\delta$ can not be too small as is required by the latest torsion-balance experiment [16]. Constraints from cosmology also lead to non-trivial bounds on extra dimensions $[17,18]^{1}$. So, we take $\delta \in[2,6]$ to do study its effect to the hadronic production.

As is shown in Eq.(11), PDFs $F_{H_{1}, P_{1}}^{i}\left(x_{1}, \mu_{F}^{2}\right)$ and $F_{H_{2}, P_{2}}^{j}\left(x_{2}, \mu_{F}^{2}\right)$ generate certain uncertainties in the estimation. PDFs are of non-perturbative nature, and in Eq.(1) they have been factorized out at the energy scale $\mu_{F}^{2}$ with the help of pQCD factorization theorem. The PDFs can be obtained only through global fitting of the experimental data and evolute them to the requested characteristic scale in standard way of $\mathrm{pQCD}$, so there are several groups, e.g. CTEQ [21], GRV [22] and MRS [23] etc, who devote themselves to offer accurate PDFs to the world, and to keep PDFs updated with the newly available relevant experimental data. Thus in literature, different versions of PDFs (including different issues by the same group) are used in the estimates of the hadronic production. To be self-consistent with the LO pQCD calculation, we shall adopt the two LO PDFs: CTEQ6L [21] and MRST2001L[23] as typical examples for PDFs. The versions of the gluon distributions ended with ' $\mathrm{L}$ ' are accurate up-to the leading logarithm order (LLO), i.e., their QCD evolution effects with $\alpha_{s}$ running are included, so for the production to show the uncertainties correctly up to LO accuracy, it is necessary for the PDFs, the hard subprocess and the QCD 'coupling constant'

\footnotetext{
${ }^{1}$ By using a hyperbolic curved other than the flat extra dimensions [19], it has been argued that those cosmology constraints can be naturally satisfied [20].
} 
$\alpha_{s}$ 'run' to the energy scale $Q^{2}$ properly. When computing the production and taking the PDFs from one version of the three groups, the running $\alpha_{s}$ should also be taken from the same group.

As for the leading order estimation, how to choose the energy scale $Q^{2}$ is a very tricky problem. If $Q^{2}$ is chosen properly, the results may be quite accurate. From experience, for a hard subprocess with two-body final state, generally the choice of $Q^{2}=\hat{s} / 4$ can lead to an accurate LO result. To see the uncertainty caused by different choices of $Q^{2}$, we take two typical types for $Q^{2}$, i.e. Type $A: Q^{2}=\hat{s} / 4$ with $\hat{s}$ the squared center of mass energy of the subprocess; Type $B: Q^{2}=M_{t}^{2} \equiv p_{t}^{2}+m^{2}$, the squared transverse mass of the graviton $/ Z^{0}$ respectively. And further more, To see the uncertainties from $Q^{2}$ choice, instead of variation on the choices with $Q^{2}=\mu_{F}^{2}$, the authors in literature, such as Ref.[24], also try $Q^{2} \neq \mu_{F}^{2}$ and see the uncertainty. Here following them, we also calculate the distributions with $Q^{2} \neq \mu_{F}^{2}$. More explicitly, as suggested in Refs. [24, 25], we take $\mu_{F}^{2} \in\left[M_{t}^{2} / 4,4 M_{t}^{2}\right]$ and $Q^{2} \in\left[M_{t}^{2} / 4,4 M_{t}^{2}\right]$ simultaneously to do the discussion.

Other parameters like $m_{Z^{0}}, x_{W}$ and the fraction of $Z^{0}$ decaying to invisible particles affect the production slightly, so we directly take them to be their center values as adopted in the literature, e.g. $m_{Z^{0}}=91.187 \mathrm{GeV}, x_{w}$ to be 0.2311 and the fraction of $Z^{0}$ decaying to invisible particles like (anti)neutrinos $\nu(\bar{\nu})$ to be the center value of $(20.00 \pm 0.06) \%$ [15].

Another important thing we need to be careful is the regulation of the cross section to avoid the non-perturbative regime, i.e. to deal with the signatures arising from collisions with parton center-of-mass energies of order $M_{D}$ or above properly. At $\sqrt{\hat{s}} \gg M_{D}$, parton collisions are expected to produce classical black holes [26]. As has been pointed out that the discrepancy between the two cases with or without the cut $\hat{s} \leq M_{D}^{2}$ shall be increased with the increment of $E_{T, j e t}^{\text {min }}$ and decreased with the increment of $M_{D}$ [12], therefore by taking proper $E_{T, j e t}$ cut in experimental analysis, one can select different ranges of $M_{D}$ to probe perturbatively. And numerically, we can find that under the present conditions, with or without the cut $\hat{s} \leq M_{D}^{2}$ shall affect our final conclusions slightly, so we shall adopt the cut $\hat{s} \leq M_{D}^{2}$ in all the following discussions. 




FIG. 1: Total jet+nothing cross-section at LHC integrated for the requirement that $E_{T, j e t}>E_{T, j e t}^{\text {min }}$ with an acceptance cut on the jet rapidity $|\eta| \leq 3$. The dash-dotted line is SM background, the solid, dashed and dotted lines are for $\delta=2,4$ and 6 respectively.

\section{A. The uncertainties in estimates}

Firstly, we show the uncertainty caused by the different choice of extra dimensions $\delta$. For the purpose, we fix PDF to be CTEQ6L, $M_{D}=5 T e V, Q^{2}=\hat{s} / 4$ and $\mu_{F}^{2}=Q^{2}$. We show the signal and the background rates for the transverse jet energy larger than $E_{T, j e t}^{\min }$ in FIG 1, with an acceptance cut on the jet rapidity $|\eta| \leq 3$. It is shown that the signal rates decrease with the increment of $\delta$. The SM background is bigger than the graviton signal in the lower transverse jet energy region but it drops down much quickly than the signal, and one may distinguish the signal from the background in large transverse jet energy region. In another words, the large transverse jet energy region shall provide an effective platform to distinguish the signal and the background.

Secondly, we show the uncertainty from different choices of $Q^{2}$ and $\mu_{F}^{2}$. For such purpose, we fix PDF to be CTEQ6L, $M_{D}=5 T e V, \delta=4$. It is found that by taking two choices of $Q^{2}$ (type A and type B) under the case of $\mu_{F}^{2}=Q^{2}$, the uncertainties for both the background and the signal are small, i.e. the differences between these two types of $Q^{2}$ are less than $10 \%$ for both the background and the signal. Further more, we show the case of $\mu_{F}^{2} \neq Q^{2}$ by shaded band in FIG 2 , with an acceptance cut on the jet rapidity $|\eta| \leq 3$, where $\mu_{F}^{2} \in\left[M_{t}^{2} / 4,4 M_{t}^{2}\right]$ and $Q^{2} \in\left[M_{t}^{2} / 4,4 M_{t}^{2}\right]$. It is found the largest value is obtained 


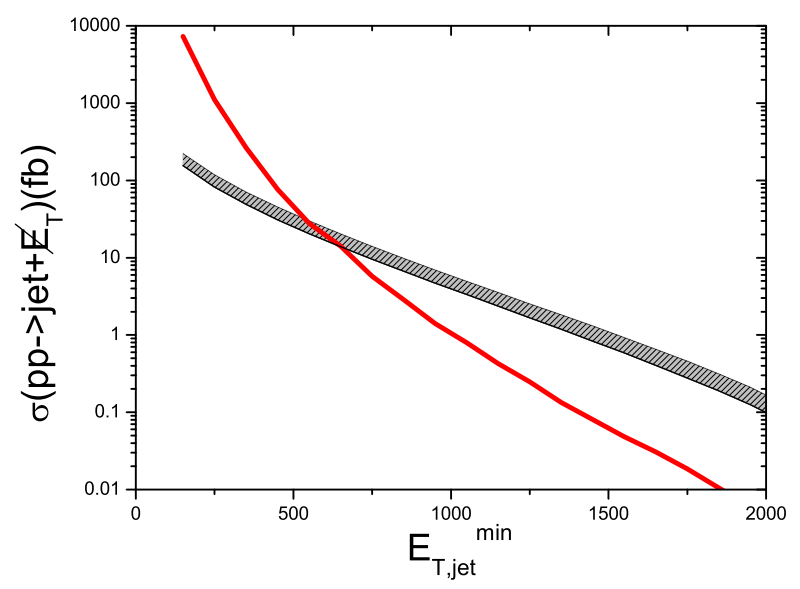

FIG. 2: Total jet+nothing cross-section at LHC integrated for the requirement that $E_{T, j e t}>E_{T, j e t}^{\text {min }}$ with an acceptance cut on the jet rapidity $|\eta| \leq 3$. The thinner and thicker shaded bands stand for the SM background and the signal respectively, with the upper edge for $\mu_{F}^{2}=M_{t}^{2} / 4$ and $Q^{2}=M_{t}^{2} / 4$, and lower edge for $\mu_{F}^{2}=4 M_{t}^{2}$ and $Q^{2}=4 M_{t}^{2}$.

TABLE I: Total jet+nothing cross-section $\sigma\left(p p \rightarrow j e t+E_{T, j e t}\right)$ (in unit: fb) versus PDFs at LHC integrated for the requirement that $E_{T, j e t}>E_{T, j e t}^{\text {min }}$ with an acceptance cut on the jet rapidity $|\eta| \leq 3$. Two LO PDFs: CETQ6L and MRST2001L, and one NLO PDF: CTEQ6M, are adopted.

\begin{tabular}{|c||c|c|c||c|c|c||}
\hline \multicolumn{1}{|c||}{} & \multicolumn{3}{c||}{ Signal with $E_{T, j e t}>E_{T, \text { jet }}^{\text {min }}$} & \multicolumn{2}{c||}{ SM background with $E_{T, j e t}>E_{T, j e t}^{\text {min }}$} \\
\hline PDFs & $0.50 \mathrm{TeV}$ & $1.0 \mathrm{TeV}$ & $1.5 \mathrm{TeV}$ & $0.50 \mathrm{TeV}$ & $1.0 \mathrm{TeV}$ & $1.5 \mathrm{TeV}$ \\
\hline \hline CTEQ6L & 32. & 5.1 & 0.92 & 46. & 1.1 & 0.070 \\
\hline MRST2001L & 31. & 4.5 & 0.73 & 47. & 1.0 & 0.060 \\
\hline \hline CTEQ6M & 40. & 6.3 & 1.1 & 57. & 1.1 & 0.074 \\
\hline \hline
\end{tabular}

when $\mu_{F}^{2}=M_{t}^{2} / 4$ and $Q^{2}=M_{t}^{2} / 4$, and the lowest value is obtained when $\mu_{F}^{2}=4 M_{t}^{2}$ and $Q^{2}=4 M_{t}^{2}$. And from FIG.2 one may also observe that while the uncertainty for the background changes to be around $20 \%$ (as shown by the thinner shaded band), the uncertainty for the signal can be changed up to 50\% (as shown by the thicker shaded band).

Thirdly, we show the uncertainty from different choices of PDFs by fixing $M_{D}=5 T e V$, 




FIG. 3: Total jet+nothing cross-section at LHC integrated for the requirement that $E_{T, j e t}>E_{T, j e t}^{\min }$ with an acceptance cut on the jet rapidity $|\eta| \leq 3$. Two typical LO PDFs, e.g. CTEQ6L, and MRST2001L are adopted.

$\delta=4$ and $\mu_{F}^{2}=Q^{2}=\hat{s} / 4$. We show the results for two LO PDFs in FIG 3 with an acceptance cut on the jet rapidity $|\eta| \leq 3$, i.e. CTEQ6L and MRST2001L. It is found that the results of CTEQ6L and MRST200L are close to each other, i.e the difference is less than 20\%. More explicitly, we show the total jet+nothing cross-section $\sigma\left(p p \rightarrow j e t+E_{T, j e t}\right)$ versus PDFs at LHC in TAB, TAB, Thows more explicitly that even though the SM background is bigger than the graviton signal in the lower transverse jet energy region but it drops down quickly, and at $E_{T, j e t}^{\text {min }} \sim 1 T e V$ it is less than $25 \%$ of the signal. At the present the next-to-leading order (NLO) results are not available ${ }^{2}$, and to have a rough idea on how NLO calculation will affect the present results, we take the 'miss matched' NLO PDF as CTEQ6M [21] to do the calculation. And it is found that the results from the "miss marched' CTEQ6M shall be bigger than that of CTEQ6L by about $25 \%$. So a full NLO estimation shall be helpful to improve our present estimations.

Finally, we show the uncertainty from different choices of $M_{D}$ by fixing PDF to be CTEQ6L, $\delta=4, Q^{2}=\hat{s} / 4$ and $\mu_{F}^{2}=Q^{2}$. We show the signal and the background rates for the transverse jet energy larger than $E_{T, j e t}^{\text {min }}$ in FIG.4 with an acceptance cut on the jet rapidity $|\eta| \leq 3$. It is shown that the rate decreases with the increment of $M_{D}$ under the

${ }^{2}$ Such a NLO calculation is in progress $[27]$. 


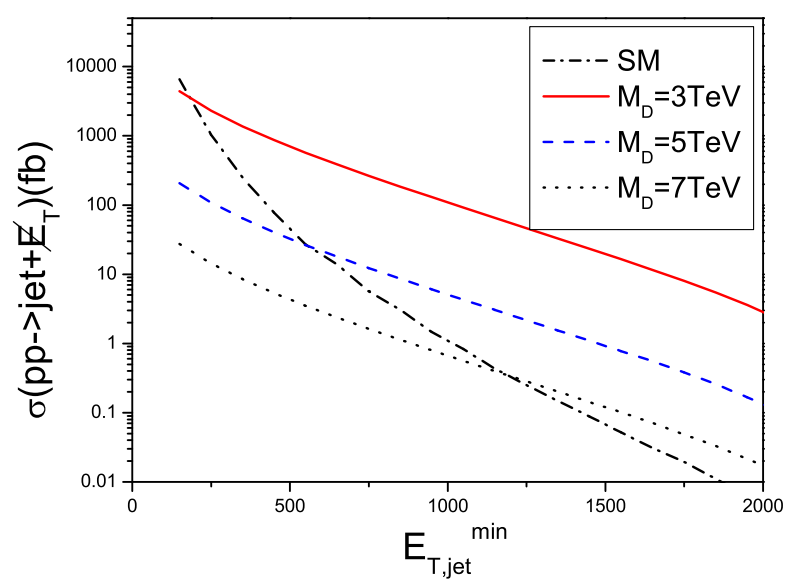

FIG. 4: Total jet+nothing cross-section at LHC integrated for the requirement that $E_{T, j e t}>E_{T, j e t}^{\min }$ with an acceptance cut on the jet rapidity $|\eta| \leq 3$. Three typical choices for $M_{D}=3 \mathrm{GeV}, 5 \mathrm{GeV}$ and $7 \mathrm{GeV}$ are adopted.

TABLE II: Corresponding $M_{D}$ sensitivity ranges versus $\delta$ with the high integrated luminosity $\mathcal{L}=100 \mathrm{fb}^{-1} . M_{D}(3 \sigma)$ stands for the $3 \sigma$ exclusion limit and $M_{D}(5 \sigma)$ stands for $5 \sigma$ observable limit, where the center values are obtained by taking CTEQ6L, $Q^{2}=\hat{s} / 4$ and $\mu_{F}^{2}=Q^{2}$ and the errors are caused by the above mentioned main uncertainty sources.

\begin{tabular}{|c||c|c||}
\hline$\delta$ & $M_{D}(3 \sigma-$ exclusion $)$ & $M_{D}(5 \sigma-$ observable $)$ \\
\hline \hline 2 & $9.2 \pm 0.7 \mathrm{TeV}$ & $8.8 \pm 0.9 \mathrm{TeV}$ \\
\hline 4 & $6.1 \pm 0.5 \mathrm{TeV}$ & $5.9 \pm 0.5 \mathrm{TeV}$ \\
\hline 6 & $5.3 \pm 0.3 \mathrm{TeV}$ & $5.1 \pm 0.3 \mathrm{TeV}$ \\
\hline \hline
\end{tabular}

same condition $E_{T, j e t}>E_{T, j e t}^{\text {min }}$.

\section{B. To what energy scale can LHC probe ?}

It is found that the SM background changes slightly within the reasonable regions of the above mentioned uncertain sources, so it can be treated as a basis to decide to what energy scale can LHC probe. Since the large extra dimensions can be probed only when the 
deviation of the cross section within the framework of large extra dimension model from the SM background is large enough, we adopt the $5 \sigma$ large extra dimension effect observable limit and $3 \sigma$ exclusion limit as suggested in the literature [14] to extract the constraint of fundamental energy scale $M_{D}$, i.e.

$$
\begin{aligned}
& \Delta \sigma=\sigma_{L E D}-\sigma_{B g d} \geq \frac{5 \sqrt{\sigma_{L E D} \mathcal{L}}}{\mathcal{L}} \\
& \Delta \sigma=\sigma_{L E D}-\sigma_{B g d} \leq \frac{3 \sqrt{\sigma_{L E D} \mathcal{L}}}{\mathcal{L}} .
\end{aligned}
$$

The corresponding $M_{D}$ sensitivity ranges versus $\delta$ are shown in TAB $\llbracket$ with high integrated luminosity $\mathcal{L}=100 \mathrm{fb}^{-1}$, where the cuts $|\eta| \leq 3$ and $E_{T, j e t}>1.0 \mathrm{TeV}$ are adopted. It has found that with integrated luminosity $\mathcal{L} \sim$ several $f b^{-1}$, the main uncertainty comes from the instrumental background [6, 7, 8, 9], which includes both the systematic and the statistical errors. So we have taken a higher integrated luminosity $\mathcal{L}=100 \mathrm{fb}^{-1}$ to do our calculation such that the systematic error is dominant. And in doing the calculation, we require that $\sigma_{L E D}>\sigma_{B k g d}$, since it has been found that the present adopted effective gravity theory is mostly reliable in this region as shown in Ref.[12]. It is found that the effective scale $M_{D}$ can be probed up to $(8.8 \pm 0.9) \mathrm{TeV}$ for $\delta=2,(5.9 \pm 0.5) \mathrm{TeV}$ for $\delta=4$ and $(5.1 \pm 0.3) \mathrm{TeV}$ for $\delta=6$, where the center values are obtained by taking CTEQ6L, $Q^{2}=\hat{s} / 4$ and $\mu_{F}^{2}=Q^{2}$ and the errors are caused by the above mentioned main uncertainty sources that varies within their reasonable regions accordingly.

Further more, one may observe that the center values for $M_{D}$ decreases with the increment of $\delta$. Our present results for $M_{D}$ versus $\delta$ with $5 \sigma$ observable limit as shown in TAB!I are consistent with the values of maximum $M_{D}$ determined in Ref.[12] (TAB.3 there for the same integrated luminosity $\mathcal{L}=100 \mathrm{fb}^{-1}$ ) within reasonable uncertainties, with the center value of our present one slightly bigger than that of Ref.[12], which is mainly caused by the fact that different criterion was adopted in Ref.[12], i.e. a fixed systematic error that is about $10 \%$ is adopted to do the discussion.

It has been argued that [12] if the discrepancy for the results with or without the cut $\hat{s} \leq M_{D}^{2}$ becomes larger, then the ultraviolet contributions become important, and then our present estimation may be not under control. Numerically, we find that such discrepancy is small for $\delta \leq 4$ (e.g. it is less than $1 \%$ for $\delta=2$ and $10 \%$ for $\delta=4$ ) by taking the $M_{D}$ values listed in TAB $\llbracket$ while for even larger $\delta$ such discrepancy becomes quite large, e.g. for $\delta=6$ such discrepancy is up to $100 \%$ by taking $M_{D}=5.1 \mathrm{TeV}$. This shows that our present 
TABLE III: Maximum $M_{D}$ sensitivity versus $\delta$ with the low integrated luminosity $\mathcal{L}=10 \mathrm{fb}^{-1}$. The center values are obtained by taking CTEQ6L, $Q^{2}=\hat{s} / 4$ and $\mu_{F}^{2}=Q^{2}$ and the errors are caused by the above mentioned main uncertainty sources.

\begin{tabular}{|c||c|c|c||}
\hline$\delta$ & 2 & 4 & 6 \\
\hline \hline $\operatorname{Max} M_{D}$ & $8.2 \pm 0.5 \mathrm{TeV}$ & $5.7 \pm 0.3 \mathrm{TeV}$ & $4.9 \pm 0.2 \mathrm{TeV}$ \\
\hline
\end{tabular}

adopted effective theory may not be fully applicable for such a large extra dimension. One may hope to decrease such discrepancy by lowering the value of $E_{T, j e t}^{\min }$ since a smaller $E_{T, j e t}^{\min }$ leads to a smaller discrepancy, however by doing this, the probed $M_{D}$ sensitivity range shall only be slightly lowered due to the fact that $E_{T, j e t}^{\text {min }}$ can not be set too small otherwise it will be more difficult to distinguish the signal from the background, as has been shown in the last subsections that the background shall increase much more quickly than the signal with a decreasing $E_{T, j e t}^{\min }$.

It may be also interesting to make a discussion on the maximum $M_{D}$ sensitivity with smaller integrated luminosity, e.g. $10 \mathrm{fb}^{-1}$. Under such case both symmetric and statistical errors are comparable, and now the criterions (11,12) are not applicable, so we adopt the criterion suggested by Ref.[12] to do the discussion, i.e. we add the two errors in quadrature and require

$$
\sigma_{L E D}>\sqrt{2} \frac{5 \sqrt{\sigma_{B g d} \mathcal{L}}}{\mathcal{L}}
$$

The corresponding $M_{D}$ sensitivity ranges versus $\delta$ are shown in TAB $\underline{\text { III }}$ with lower integrated luminosity $\mathcal{L}=10 \mathrm{fb}^{-1}$, where the cuts $|\eta| \leq 3$ and $E_{T, j e t}>1.0 \mathrm{TeV}$ are adopted and the errors are caused by the above mentioned main uncertainty sources that varies within their reasonable regions accordingly.

\section{SUMMARY}

It is found that with sufficient luminosity at LHC the fundamental gravity scale can be probed up to several $\mathrm{TeV}$, with the precise value depending on the number of extra dimensions. In the present paper, we have presented a detailed discussion on the leading experimental signal of real graviton at LHC based on the process $p p \rightarrow j e t+\mathbb{E}_{T}$ with the 
help of the quantum gravity theory with large extra dimensions. The main standard model background to these processes together with their uncertainties have also been discussed. It is found that in higher transverse jet energy region, e.g. $E_{T, j e t}>1.0 \mathrm{TeV}$, and with certain number of extra dimensions, the quantum gravity signal can be distinguished from the background.

Acknowledgements: We would like to thank Prof. F.Y. Li for helpful discussions. This work was supported in part by Natural Science Foundation Project of CQ CSTC under grant number 2008BB0298 and Natural Science Foundation of China under grant number 10805082, and by the grant from the Chinese Academy of Engineering Physics under the grant numbers: 2008T0401 and 2008T0402.

[1] N. Arkani-Hamed, S. Dimopoulos and G.R. Dvali, Phys.Lett. B429, 263(1998).

[2] I. Antoniadis, N. Arkani-Hamed, S. Dimopoulos and G.R. Dvali, Phys.Lett. B436, 257(1998).

[3] G. Landsberg, in representation of CDF Collaboration and D0 Collaboration, hep-ex/0412028.

[4] T.G. Rizzo, Phys.Rev. D59, 115010(1999).

[5] Y. Uehara, Mod.Phys.Lett. A17, 1551(2002).

[6] T. Aaltonen et al. [CDF Collaboration], arXiv: 0807.3132[hep-ex].

[7] V.M. Abazov et al. [Do Collaboration], Phys.Rev.Lett. 101, 011601(2008).

[8] S.S. Yu [CDF Collaboration and D0 collaboration], arXiv:0807.3523[hep-ex].

[9] S. Shmatov [CMS Collaboration], Nucl.Phys.Proc.Suppl. 177, 330(2008).

[10] M. Perelstein, arXiv:0809.1843[hep-ph].

[11] T. Han, J.D. Lykken and R.J. Zhang, Phys.Rev. D59, 105006(1999).

[12] G.F. Giudice, R. Rattazzi and J.D. Wells, Nucl.Phys. B544, 3(1999).

[13] E.A. Mirabelli, M. Perelstein and M.E. Peskin, Phys.Rev.Lett. 82, 2236(1999).

[14] H. Sun, R.Y. Zhang, P.J. Zhou, W.G. Ma, Y. Jiang and L. Han, Phys.Rev. D71, 075005(2005).

[15] C. Amsler et al. (Particle Data Group), Phys.Lett. B667, 1(2008).

[16] D.J. Kapner et al., Phys.Rev. Lett.98, 021101(2007).

[17] L.J. Hall and D. Smith, Phys.Rev. D60, 085008(1999); V.D. Barger, T. Han, C. Kao and R.J. 
Zhang, Phys.Lett. B461, 34(1999).

[18] Steen Hannestad and Georg G. Raffelt, Phys.Rev. D67, 125008(2003); Erratum-ibid. D69, 029901(2004).

[19] N. Kaloper, J. March-Russell, G.D. Starkman and M. Trodden, Phys.Rev. Lett.85, 928(2000).

[20] G.D. Starkman, D. Stojkovic and M. Trodden, Phys.Rev. Lett.87, 231303(2001); H. Melbeus and T. Ohlsson, JHEP 0808, 077(2008).

[21] H.L. Lai, et al., JHEP0207, 012(2002).

[22] M. Glueck, E. Reya and A. Vogt, Eur. Phys. J.C5, 461(1998).

[23] A.D. Martin, R.G. Roberts, W.J. Stirling and R.S. Thorne, Eur. Phys. J. C23, 73(2002).

[24] M. Klasen, B.A. Kniehl, L.N. Mihaila and M. Steinhauser, Phys. Rev. Lett. 89, 032001(2002).

[25] C.H. Chang, J.X. Wang and X.G. Wu, Phys.Rev. D70, 114019(2004).

[26] D. Bourilkov, [ATLAS and CMS Collaboration], arXiv: 0807.4960[hep-ex]; M. Cavaglia, R. Godang, L.M. Cremaldi and D.J. Summers, JHEP0706, 055(2007).

[27] X.G. Wu et al., The next-to-leading order estimation of the monojet graviton signal at LHC, in preparation. 